\title{
Investigation of Four-Wavelength Regenerator Using Polarization- and Direction-Multiplexing
}

\author{
L. Provost, F. Parmigiani, P. Petropoulos, D. J. Richardson, K. Mukasa, M. Takahashi, J. Hiroishi, and \\ M. Tadakuma
}

\begin{abstract}
We demonstrate a four-channel extension to the Mamyshev regenerator and investigate its potential for the simultaneous processing of $4 \times 10 \mathrm{~Gb} / \mathrm{s}$ return-to-zero wavelength division-multiplexed channels. Mitigation of the interchannel nonlinearities is achieved through the rapid walkoff induced by both the large differential group delay of a specially developed polarization maintaining highly nonlinear fiber and a bidirectional architecture. Numerical and experimental results demonstrate the reduction of interchannel crosstalk as well as the potential of the scheme for $2 \mathrm{R}$ regeneration.
\end{abstract}

Index Terms-All-optical regeneration, nonlinear optical fiberbased devices, optical Kerr effect, polarization multiplexing, selfphase modulation.

\section{INTRODUCTION}

$\mathbf{F}$ UTURE optical networks are expected to incorporate a significant degree of all-optical processing in order to overcome the inherent bandwidth limitations associated with electronics and associated optoelectronic conversion. Over the years, much work has been reported on the development of all-optical techniques for critical basic functions such as wavelength conversion, signal monitoring, and regeneration. To date, most of these studies have concerned single channel devices with little consideration of the prospects for multiwavelength operation. However, it is clear that if it is possible to process multiple channels within a single device, then this could offer major practical and economic advantages for any real-world system. All-optical regeneration is a particularly desirable and enabling network function. Fiber-based regeneration represents one of the most promising technical approaches due to the ultrafast nonlinear response and the design versatility. However, a direct extension of the existing single-channel techniques to the multichannel regime is fundamentally restricted by interchannel nonlinearities. For example, the well-known Mamyshev regenerator approach [1] which relies on self-phase modulation (SPM) and subsequent offset filtering, is ordinarily compromised by cross-phase modulation (XPM) (and to a

Manuscript received April 8, 2008; revised July 11, 2008. First published August 22, 2008; current version published September 26, 2008. This work was supported in part by the European Union STREP under Project TRIUMPH (IST027638).

L. Provost, F. Parmigiani, P. Petropoulos, and D. J. Richardson are with the Optoelectronics Research Centre, University of Southampton, Southampton SO17 1BJ, U.K. (e-mail: lap@ orc.soton.ac.uk).

K. Mukasa, M. Takahashi, J. Hiroishi, and M. Tadakuma are with the Furukawa Electric Company, Fitel Photonics Laboratory, Ichihara, Japan.

Color versions of some of the figures in this letter are available online at http:// ieeexplore.ieee.org.

Digital Object Identifier 10.1109/LPT.2008.2003389 lesser extent four-wave mixing), as soon as additional copropagating channels are introduced within the same fiber. These nonlinear effects compete directly with the SPM, resulting in the generation of strong distortions in the broadened spectra which manifest themselves as large signal power fluctuations after the output filter. A substantial reduction in the channel interactions can, however, be realized by ensuring rapid and complete "walk through" of the data pulses by exploiting some form of DGD walkoff. However, so far just a few experimental studies have extended the technique to the multiwavelength regime. In this letter, mitigation of the interchannel crosstalk was obtained using specific chromatic dispersion management within fiber assemblies [2], [3], dispersion decreasing fiber [4], or bidirectional propagation [5].

In this letter, we combine bidirectional propagation with polarization multiplexing in a specially developed polarization maintaining highly nonlinear fiber (PM-HNLF) to allow processing of four wavelength division-multiplexing (WDM) channels. Experimental measurements of the system [6] are supported by numerical studies on the benefits of our scheme.

\section{PRINCIPLE OF OPERATION}

The key aspect of our work is to exploit the fast interchannel pulse walkoff due to the large differential group delay (DGD) achievable within a PM-HNLF in order to reduce the nonlinear crosstalk between orthogonally polarized channel pairs. The approach is illustrated in Fig. 1(a), where two linearly polarized optical signals are properly aligned to the two eigen-axes of the PM-HNLF used to generate the SPM. The resulting output signals are then separated and individually offset filtered. (Note that in addition to the induced-DGD walkoff a further three-fold reduction in XPM is obtained due to the tensorial nature of the Kerr effect.)

In order to investigate the influence of the absolute DGD value on the interchannel crosstalk, we numerically compared the variation in the filtered signal (channel 1) output power in the presence of an interference channel (channel 2) at five different points (A-E) on the static power transfer function (TF) of the device [see Fig. 1(b)]. The pulses for channels 1 and 2 were assumed to be transform-limited Gaussians with a full-width at half-maximum (FWHM) pulse duration of $8.3 \mathrm{ps}$. Channel 2 comprised a $10-\mathrm{Gb} / \mathrm{s}$ signal of alternating marks and spaces. A maximum peak power of $1.5 \mathrm{~W}$ was assumed. Channel 2 was characterized by an initial temporal delay $\tau$ relative to channel 1 at the fiber input, and its central frequency was offset by $600 \mathrm{GHz}$ from Channel 1. The fiber properties used correspond to those reported in the experimental section of this letter. Fig. 1(c) shows the output power variation 
(a)

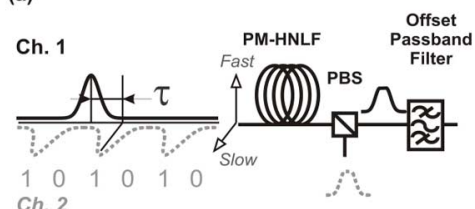

(c)

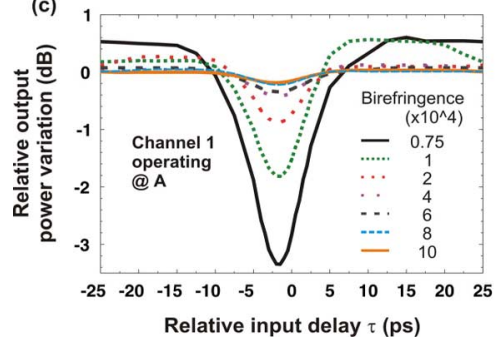

(b)
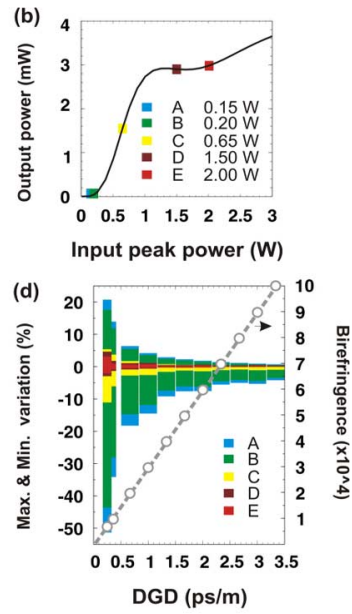

Fig. 1. (a) Principle of operation. (b) Static power TF for Channel 1 as a function of input power for points A-E. (c) Influence of birefringence on the TFs variation obtained at point $\mathrm{A}$ as a function of the initial delay $\tau$ (pulse peak power of Ch. $2=1.5 \mathrm{~W}$ ). (d) TF relative variation extrema as a function of DGD at various input powers and corresponding values of birefringence.

experienced for a channel 1 input power of $0.15 \mathrm{~W}$ (point A on the TF) normalized relative to the single channel case as a function of $\tau$ for different birefringence values. This low signal power level was chosen for this initial study since the impact of the nonlinear crosstalk is expected to be more pronounced on the spaces rather than marks [as confirmed in Fig. 1(d)]. The variations clearly demonstrate a crosstalk dependence on the initial delay, especially at low birefringence values. However, as the birefringence is progressively increased, the peak-to-peak variations as a function of $\tau$ are minimized and tend to the single channel case as desired. In Fig. 1(d), we show the corresponding peak-to-peak variations for points A-E on the TF as the total DGD is changed. It is clear that increasing the DGD decreases the sensitivity to XPM. A minimum DGD value of $\sim 1.3 \mathrm{ps} / \mathrm{m}$ is required to limit the XPM perturbations to within $+/-10 \%$ at low input powers (A-B), whereas at high input powers (D-E), the influence of the DGD is less significant (a minimum DGD value of $\sim 1 \mathrm{ps} / \mathrm{nm}$ is sufficient to ensure that SPM dominates the XPM). Note that the computations are idealized since no source of polarization mixing or linear crosstalk were considered. Moreover, in the proposed configuration the total DGD value is predominantly determined by the fiber birefringence since the contribution from the relative group velocity difference (GVD) is only of the order of $\sim 0.02 \mathrm{ps} / \mathrm{m}$ for a $600-\mathrm{GHz}$ channel separation. Although the effects of XPM are not completely eliminated at low input powers, a DGD value of the order of $\sim 1.5 \mathrm{ps} / \mathrm{m}$ appears to be sufficient to provide resilience to interchannel crosstalk and to provide output characteristics similar to those of the single channel case.

\section{EXPERIMENTAL SETUP}

By implementing this approach in a bidirectional architecture [5], simultaneous processing of four WDM channels is possible. The corresponding experimental setup is reported in Fig. 2 [6]. Four 10-GHz pulsed data streams were generated by gain-switched laser diodes with pulse durations of 7-8 ps. All channels were individually amplitude modulated with

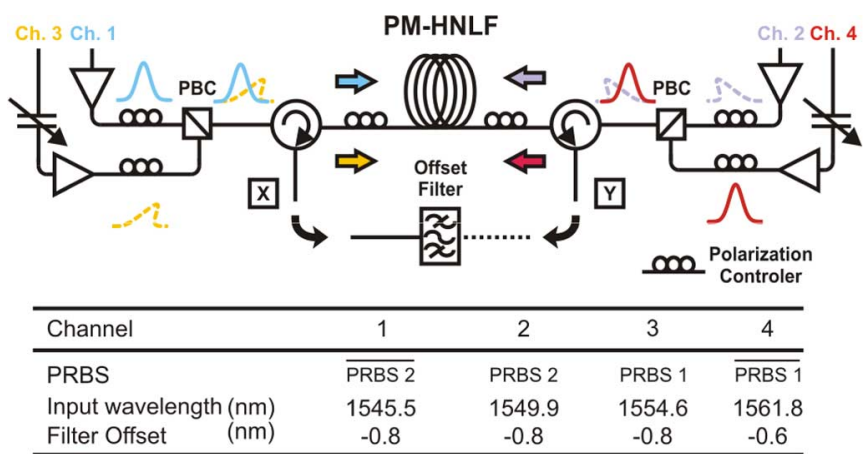

Fig. 2. Experimental setup and corresponding channel properties.

two $2^{31}-1$-long pseudorandom bit sequences (PRBS). The corresponding channel carrier frequency and PRBS allocation are reported in Fig. 2. Each channel was then individually amplified and fed into the PM-HNLF via a polarization beam combiner (PBC). Two optical circulators separated the transmitted and the reflected signals from the HNLF at both fiber ends. Finally, a $0.53-\mathrm{nm}$ bandwidth tunable optical filter at port 3 of the circulator acted as the reshaping and decision element (see Fig. 2 for the corresponding relative offsets). The PM-HLNF was 1-km long and has a chromatic dispersion value of $-4.2 \mathrm{ps} / \mathrm{nm} / \mathrm{km}$ at $1550 \mathrm{~nm}$ (slow axis) for a nonlinear coefficient of $\sim 20 \mathrm{~W}^{-1} \cdot \mathrm{km}^{-1}$. The birefringence value was as high as $\sim 7 \times 10^{-4}$ corresponding to a DGD value of $\sim 2.3 \mathrm{ps} / \mathrm{m}$. The fiber has a loss of $4.2 \mathrm{~dB} / \mathrm{km}$ and a nominal polarization extinction ratio of $-30 \mathrm{~dB}$ (over $100 \mathrm{~m}$ ).

A slight difference in the spectral broadening was observed between the two axes of the fiber under single channel operation which we attribute to the opto-geometrical/loss anisotropy existing between the two polarization axes. This variation was compensated for by adjusting the input power accordingly. Due to the lack of sufficient high power amplifiers in our laboratory, we reduced the filter detuning for Channel 4 slightly and allowed a small power penalty for Channel 2 , thereby permitting use of lower power amplifiers within the system.

\section{RESULTS}

First, we assessed the device characteristics in terms of the static power TFs for each channel propagating in isolation. As reported in Fig. 3, the four channels exhibit somewhat different TFs, which originates predominantly from the noticeably different characteristics of the input channel pulses (spectral bandwidth, presence of side-lobes and residual chirp, etc.) and which translates into noticeably different SPM-broadened spectra (see Fig. 4). We then compared the TFs obtained in the presence of all interfering channels operating at the nominal operational input power. Optical delay lines were adjusted to ensure maximum pulse interaction between the copropagating signals. This was achieved by maximizing the generated four-wave mixing components, observed when the launched signals were copolarized. As can be seen in Fig. 3, the various TFs overlap well, illustrating the absence of strong interchannel crosstalk amongst the various channels. As already pointed out in Section II, for low input powers, we clearly observe crosstalk that manifests itself as a constant power floor and which has a different value according to the particular channel considered 


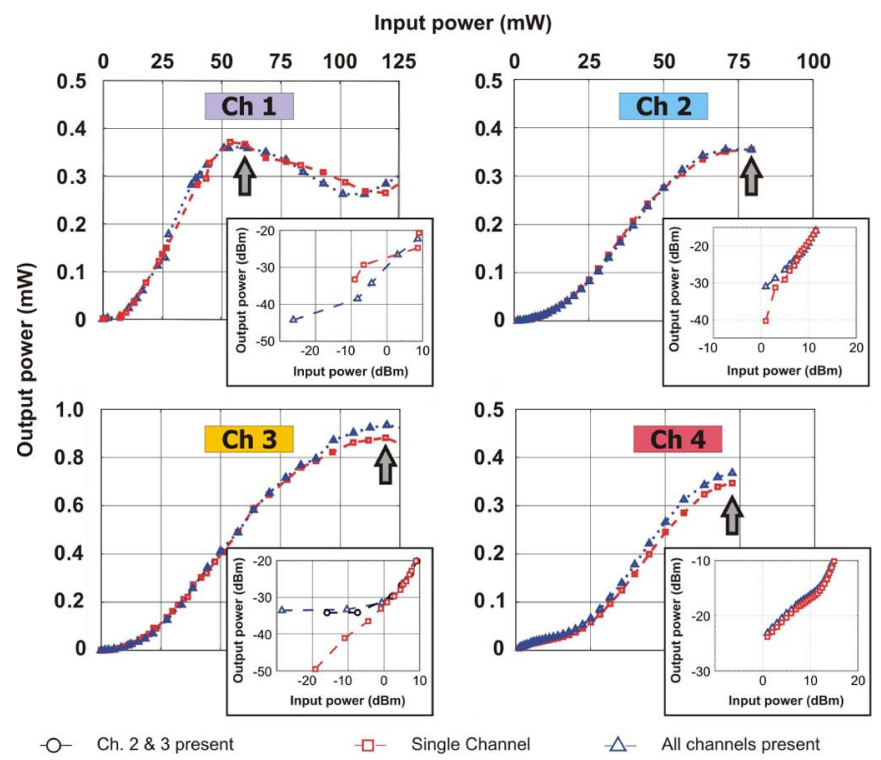

Fig. 3. Experimental TFs obtained for the four channels in both the absence and presence of the interfering channels. The arrows indicate the corresponding operating input powers. Inset graphs detail variations at low input powers $(\log -$ arithmic scale). Input powers measured at the fiber inputs.

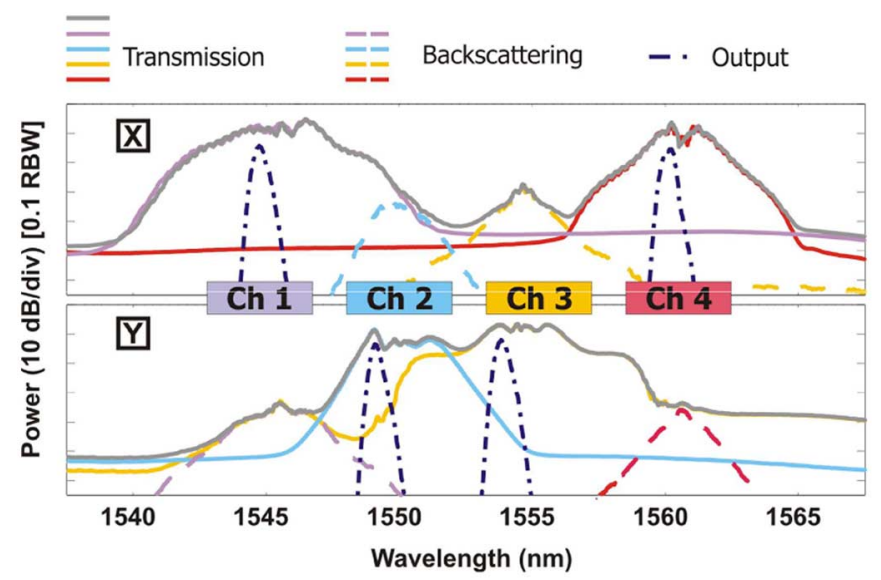

Fig. 4. Single- and multichannel spectra obtained at fiber output ports $\mathrm{X}$ and $\mathrm{Y}$ and output channels (all channels operating at nominal input operating powers).

(see inset Fig. 3). This is due to additional spectral components that fall within the offset filter bandwidth. Depending on the considered channel, this power floor originates either from the copropagating signal or from the backscattered contribution of the counterpropagating signal. A more visual illustration can be found in Fig. 4, where transmitted, backscattered, and output pulse spectra are plotted. The presence of this additional collected power results in a slight decrease in the extinction ratio of the various channels, which is primarily due to the particular channel allocation chosen.

Bit-error rate (BER) measurements were carried out for the four channels both under single- and multichannel operation (Fig. 5). As compared to the back-to-back case, our experiments confirmed that the system does not introduce any additional power penalty when operating in a single channel configuration (except for Channel 2), and similar performance is obtained when operating in the presence of the other channels. By selecting a suboptimum bias voltage setting for the modulator,

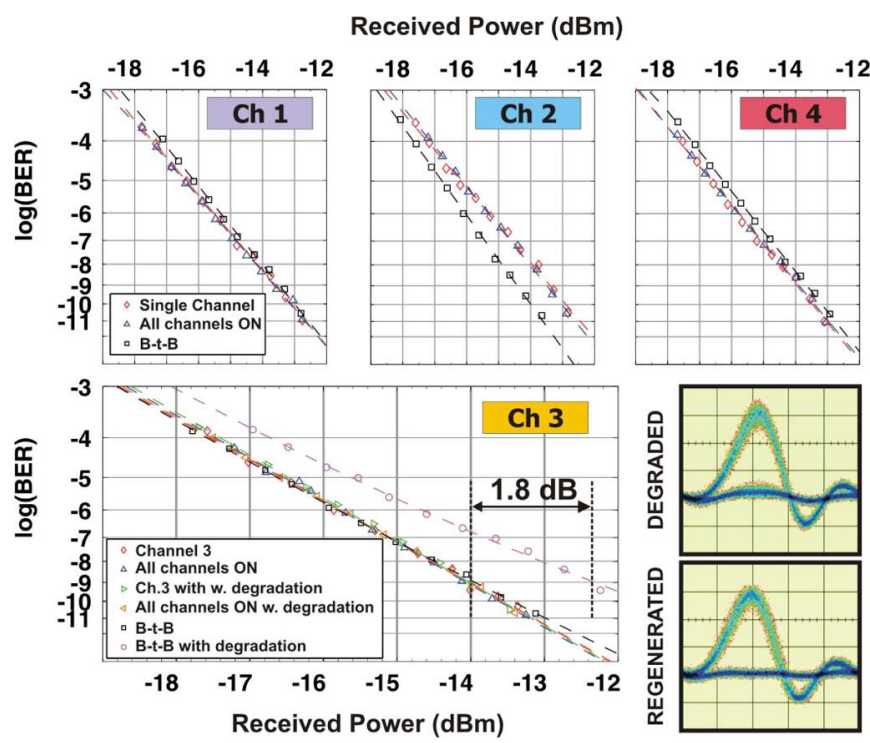

Fig. 5. BER measurements of the four channels in the absence and presence of interfering channels. The additional BER curve for Channel 3 corresponds to the degraded signal case. Corresponding eye diagrams of the input degraded and output regenerated data stream.

we then artificially degraded Channel 3 , which we believed presented the worst crosstalk scenario. A power penalty improvement of up to $\sim 1.8 \mathrm{~dB}$ (at BER $=10^{-9}$ ) can be obtained under both single and multichannel operation. The corresponding eyediagrams show both simultaneous amplitude equalization and noise rejection in the empty slot.

\section{CONCLUSION}

We have demonstrated the benefit of using a new PM-HNLF which allows simultaneous processing of $4 \times 10 \mathrm{~Gb} / \mathrm{s}$ WDM channels. The technique relies on the propagation of two channel pairs in a bidirectional configuration and uses polarization multiplexing for each of the channel pairs. Owing to the sufficiently high birefringence-induced DGD, robust tolerance to interchannel crosstalk was demonstrated. Similar TFs and BER measurements to the single channel case confirm that no additional power penalties are observed due to the presence of crosstalk originating from overlap and/or backscattering from the neighboring channels.

\section{REFERENCES}

[1] P. V. Mamyshev, "All-optical data regeneration based on self-phase modulation effect," in Proc. Eur. Conf. Optical Communications (ECOC'98), Madrid, Spain, 1998, p. 475.

[2] T. Ohara, H. Takara, S. Kawanishi, T. Yamada, and M. M. Fejer, "160-Gb/s all-optical limiter based on spectrally filtered optical solitons," IEEE Photon. Technol. Lett., vol. 16, no. 10, pp. 2311-2313, Oct. 2004.

[3] T. L. Lakoba et al., "A new robust regime for a dispersion-managed multichannel 2R regenerator," Opt. Express, vol. 15, pp. 10061-10074, 2007.

[4] D. V. Kuksenkov et al., "Nonlinear fibre devices operating on multiple WDM channels," in Proc. Eur. Conf. Optical Communications (ECOC'05), Glasgow, U.K., 2005, Paper Mo.3.5.1.

[5] L. Provost et al., "Analysis of a two-channel 2R all-optical regenerator based on a counter-propagating configuration," Opt. Express, vol. 16, no. 3, pp. 2264-2275, 2008.

[6] L. Provost et al., "Simultaneous all-optical 2R regeneration of $4 \times 10$ Gbit/s wavelength division multiplexed channels," presented at the ECOC 2007, Berlin, Germany, 2007. 